\title{
Informationen zu den Bearbeitern
}

\section{Dr. Marco Arteaga}

Rechtsanwalt Dr. Marco S. Arteaga studierte Betriebswirtschaft und Rechtswissenschaften in Oestrich-Winkel, Wiebaden, Mainz und Köln und promovierte über die Insolvenzsicherung von Unternehmer-Pensionszusagen. Er ist als Rechtsanwalt spezialisiert auf Fragen der betrieblichen Altersversorgung. Aufgrund seiner jahrzehntelangen wissenschaftlichen und praktischen Tätigkeiten auf diesem Gebiet beauftragte inn das Bundesarbeitsministerium im Jahr 2015 gemeinsam mit Prof. Dr. Peter Hanau (Universität zu Köln) mit der Weiterentwicklung der vom Ministerium zuvor veröffentlichten Vorschläge zur Schaffung von tarifvertraglich organisierter betrieblicher Altersversorgung in Deutschland. Das Gutachten wurde im April 2016 vorgestellt. Wesentliche neue Gestaltungsvorschläge daraus für die Weiterentwicklung der nur unzureichend verbreiteten betrieblichen Altersversorgung wurden in das Betriebsrentenstärkungesetz übernommen. Hierzu zählen unter anderem die garantiefreie Zielrente sowie das Optionssystem. In seiner langjährigen Berufspraxis war Dr. Arteaga geschäftsleitend in mehreren der großen Beratungsunternehmen auf dem Gebiet der betrieblichen Altersversorgung tätig. Außerdem verantwortete er auch bei mehreren Lebensversicherungsunternehmen die Geschäftsaktivitäten in der bAV.

\section{Dr. Annekatrin Veit}

Annekatrin Veit ist Rechtsanwältin und Steuerberaterin. Sie ist spezialisiert auf alle arbeitsrechtlichen, steuerrechtlichen und bilanziellen Fragestellungen zur betrieblichen Altersversorgung. Nach Stationen in Wirtschaftsprüfungsgesellschaften, Anwaltskanzleien und Beratungshäusern für betriebliche Altersversorgung ist sie bei der internationalen Rechtsanwaltskanzlei DLA Piper in München tätig. Sie berät Mandanten aller Größen und Branchen in Fragen der betrieblichen Altersversorgung, insbesondere im Rahmen von Unternehmenstransaktionen und -umstrukturierungen. Sie ist dem Fachpublikum durch zahlreiche Veröffentlichungen und Vorträge bekannt. Sie ist Mitautorin des Kommentars von Höfer/Veit/Verhuven zum Steuerrecht der betrieblichen Altersversorgung, des Kommentars zum Einkommensteuergesetz von Korn sowie der Publikation von Hanau/Arteaga/Rieble/Veit zur Entgeltumwandlung. Sie erhielt Lehraufträge der Fachhochschulen Kaiserlautern, Köln und der Universität Ulm und ist Mitglied im Fachausschuss Steuerrecht der Arbeitsgemeinschaft für betriebliche Altersversorgung (aba) sowie im Fachkreis Steuern der Arbeitsgemeinschaft Zeitwertkonten (AGZWK). Ferner gehört sie dem Eberbacher Kreis an, einem Zusammenschluss von Rechtsanwälten aus Großkanzleien, die sich auf die betriebliche Altersversorgung spezialisiert haben.

\section{Manuel Baroch Castellvi}

Rechtsanwalt Manuel Baroch Castellvi war nach anfänglicher Anwaltstätigkeit in einer renommierten Großkanzlei mehr als 20 Jahre als Syndikus in der Versicherungswirtschaft tätig. Ein Schwerpunkt lag dabei im Bereich der Lebensversicherung, insbesondere im Versicherungsvertragsrecht und im Versicherungsaufsichtsrecht. Aus dieser Tätigkeit ist er auch mit allen versicherungsbasierten Formen der betrieblichen Altersversorgung vertraut. Unter anderem begleitete er 2002 die Gründung und danach den laufenden Betrieb eines der ersten in Deutschland aktiven Pensionsfonds. Er war während seiner Unternehmenstätigkeit in diversen Verbandsgremien tätig und besitzt einen Lehrauftrag zum Versicherungsaufsichtsrecht an der Technischen Hochschule in Köln. Im Jahr 2016 wechselte er wieder in Anwaltstätigkeit zur internationalen Rechtsanwaltskanzlei DLA Piper in Köln. Dort berät er unter anderem Einrichtungen der 
betrieblichen Altersversorgung zu versicherungsaufsichtsrechtlichen und versicherungsvertragsrechtlichen Fragen. Er ist für eine Vielzahl von rechtswissenschaftlichen Veröffentlichungen verantwortlich, insbesondere zuletzt als Mitherausgeber des 2018 erschienenen Handkommentars zum VAG von Brand/Baroch Castellvi. 